\title{
The TIR instrument on TRISHNA satellite: a precursor of high resolution observation missions in the thermal infrared domain
}

Laurence Buffet, Philippe Gamet, Philippe Maisongrande, Corinne Salcedo, Philippe Crebassol

Laurence Buffet, Philippe Gamet, Philippe Maisongrande, Corinne Salcedo, Philippe Crebassol, "The TIR instrument on TRISHNA satellite: a precursor of high resolution observation missions in the thermal infrared domain," Proc.

SPIE 11852, International Conference on Space Optics - ICSO 2020, 118520Q (11 June 2021); doi: 10.1117/12.2599173

SPIE Event: International Conference on Space Optics - ICSO 2021, 2021, Online Only 


\section{International Conference on Space Optics-ICSO 2020}

Virtual Conference

30 March-2 April 2021

Edited by Bruno Cugny, Zoran Sodnik, and Nikos Karafolas
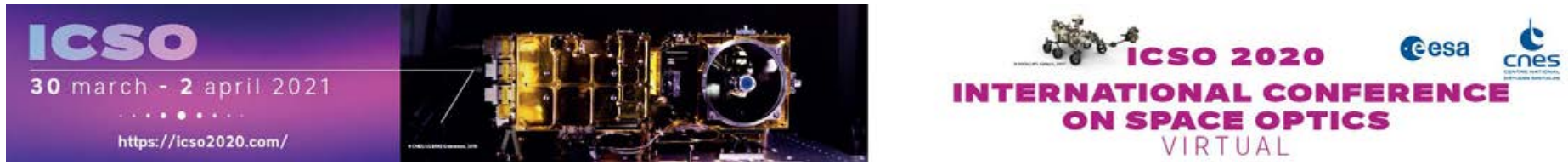

\section{The TIR instrument on TRISHNA satellite: a precursor of high resolution observation missions in the thermal infrared domain}

\section{Cesa issopreasings denes}




\title{
The TIR instrument on TRISHNA satellite: a precursor of high resolution observation missions in the thermal infrared domain
}

\author{
Laurence Buffet ${ }^{\mathrm{a}}$, Philippe Gamet ${ }^{\mathrm{a}}$, Philippe Maisongrande ${ }^{\mathrm{a}}$, Corinne Salcedo ${ }^{\mathrm{a}}$, Philippe Crebassol ${ }^{\mathrm{a}}$, \\ Renaud Binet ${ }^{\mathrm{a}}$, Aurélien Ledot ${ }^{\mathrm{a}}$, Nathalie Malechaux ${ }^{\mathrm{a}}$, Thierry Carlier ${ }^{\mathrm{a}}$, \\ Jean-Louis Roujean ${ }^{\mathrm{b}}$, Didier Charvet ${ }^{\mathrm{c}}$, François Chassat ${ }^{\mathrm{c}}$ \\ ${ }^{a}$ Centre National d'Etudes Spatiales, 18 Av. E. Belin, 31401 Toulouse cedex 9; \\ ${ }^{\mathrm{b}} \mathrm{CESBIO} ;{ }^{\mathrm{c}}$ Airbus Defence \& Space
}

\begin{abstract}
The TRISHNA program marks a step further in the fruitful cooperation built between CNES and ISRO since many years, through a new Earth observation mission dedicated to the improvement of water cycle understanding and water resource management. Thanks to its unprecedented high spatial resolution in the thermal infrared domain, together with a high revisit frequency, TRISHNA mission will significantly contribute to the detection of ecosystem stress and to the optimization of water use in agriculture in a context of global climate change.

This paper presents the TRISHNA mission context and main requirements, gives an overview of TRISHNA system architecture and describes TIR (Thermal InfraRed) instrument design with a highlight on its specific innovative features and the current development status.
\end{abstract}

Keywords: TIR, TRISHNA, thermal infrared

\section{TRISHNA MISSION}

\subsection{Context and objectives}

The TRISHNA mission (Thermal infraRed Imaging Satellite for High-resolution Natural resource Assessment) is a cooperation between the French (CNES) and Indian (ISRO) space agencies. It is intended to measure approximately twice a week the thermal infrared signal of the surface-atmosphere system globally and at $57 \mathrm{~m}$ resolution for the continents and the coastal ocean, and a resolution of $1000 \mathrm{~m}$ over deep ocean. From the global scale to much more regional scales in which decision-makers and managers are involved, water is both an essential element for life and the main vector of heat exchange in the meteorological and climate system. Today, the combined change in temperatures and rainfall regimes leads to a change in accessible freshwater reserves, $70 \%$ of which are dedicated to agricultural uses (irrigation), 25\% to industry and the rest to domestic uses.

It is therefore logical to aim for an optimized management of this resource, through a key variable, evapotranspiration. To access it, the surface temperature and its dynamics are precise indicators of the evaporation of water from soils, transpiration of plants and of the local climate.

In this context, TRISHNA and its frequent high-resolution measurements raise major scientific, economic and societal issues through the major themes that the mission addresses from the angle of research and development of applications.

Agriculture and water content of natural vegetation: the TRISHNA mission will focus on the estimation of evapotranspiration, and will contribute to the detection of water stress, the monitoring of irrigation and its management. It will provide direct information on the use of water, and in particular its agricultural consumption. These data will also indirectly contribute to water availability assessments and studies on the natural or anthropogenic causes of the variation in groundwater level. In addition, TRISHNA could be used as an early warning tool to prevent the effects of droughts on agricultural surfaces or for issues related to the health of agro-forest ecosystems, but also of wetlands and coral reefs.

In coastal areas, the deep interactions between the ocean, the atmosphere and the land generate a strong variability in the surface temperature at very fine scales. It is therefore interesting to measure the temperature of the water at the surface with high spatial and temporal precision, as this information can have several uses.

Thermal imaging with high spatial resolution and frequent observation, including night-time acquisitions will also bring key information on sea surface temperatures and winds, sub-mesoscale activity in coastal areas and in the high seas, continental waters (lakes and rivers) as well as oil spills, thermal pollutants, effluents and wastewater discharges. 
In urban areas, the increase of the frequency and intensity of heat waves expected as a consequence of climate change necessitates policies for mitigating their effects. In this context, the TRISHNA mission will provide key data for the estimation and forecasting of Urban Heat Islands and the development of comfort and/or vulnerability indexes.

Moreover, research in cryosphere science will benefit from TRISHNA measurements: snow dynamics, monitoring of glacier debris, high-altitude lake dynamics. As far as solid Earth science is concerned, monitoring of surface temperature provides information on the volcanic activity for better understanding of the processes involved through the detection of thermal anomalies.

\subsection{Mission main requirements}

A global coverage is required, with a full resolution of $57 \mathrm{~m}$ at nadir over all continental land surfaces (including inland waters) and coastal waters up to $100 \mathrm{~km}$ from the shore. The chosen orbit has a cycle of 8 days, at an altitude of $761 \mathrm{~km}$. The large swath of $+/-34^{\circ}$ gives access to a revisit frequency of at least 3 acquisitions per 8-day period, depending on the latitude as illustrated on Figure 1.

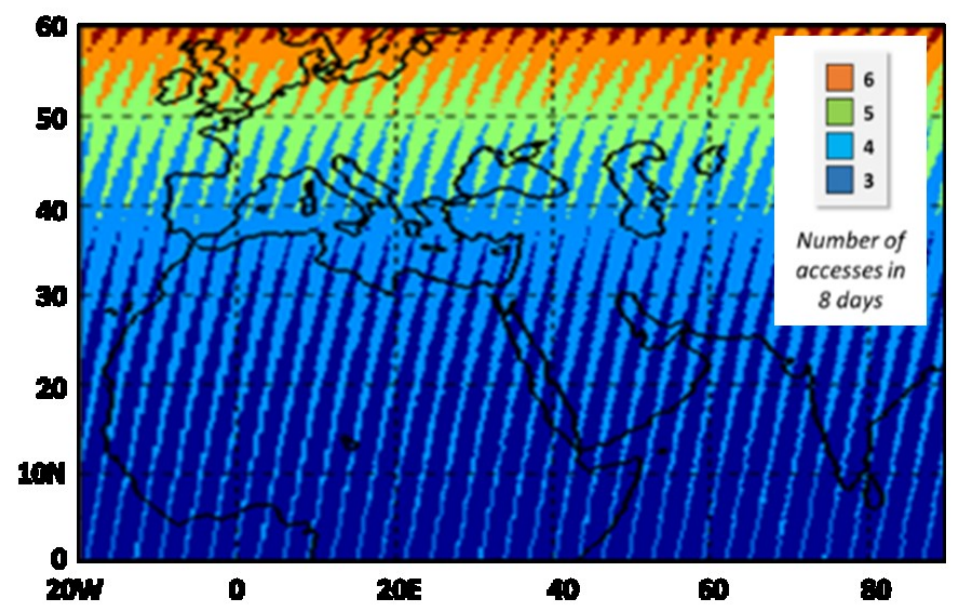

Figure 1. TRISHNA revisit frequency: number of observations per cycle of 8 days

The spectral domain covers 11 bands from visible to thermal infrared, as defined in Table 1 and Table 2, which are distributed over two instruments: the VNIR/SWIR Indian instrument and the TIR French instrument. The 4 thermal infrared bands of the TIR instrument are used for emissivity and surface temperature retrieval.

A high radiometric performance is required for the TIR instrument, with a radiometric noise better than $0.2 \mathrm{~K}$ in the most demanding bands, and an absolute radiometric calibration accuracy better than $0.5 \mathrm{~K}$.

Table 1. TRISHNA VNIR/SWIR spectral bands

\begin{tabular}{|c|c|c|c|}
\hline Band name & $\begin{array}{c}\text { Wavelength } \\
\text { Center }(\mathbf{n m})\end{array}$ & $\begin{array}{c}\text { FWHM } \\
\mathbf{( n m )}\end{array}$ & Purpose \\
\hline Blue & 485 & 70 & Detection of low clouds \\
\hline Green & 555 & 70 & Coastal, sediments, snow \\
\hline Red & 670 & 60 & Vegetation (Leaf Area Index, fCOVER, NDVI, ...) \\
\hline NIR & 860 & 40 & Vegetation (Leaf Area Index, fCOVER, NDVI, ...) \\
\hline WV & 910 & 20 & Water vapour content estimation \\
\hline Cirrus & 1380 & 30 & Detection of thin cirrus clouds \\
\hline SWIR & 1610 & 100 & AOD, snow/cloud discrimination, vgt stress, burnt areas \\
\hline
\end{tabular}


Table 2. TRISHNA TIR spectral bands

\begin{tabular}{|c|c|c|c|}
\hline Band name & $\begin{array}{c}\text { Wavelength } \\
\text { Center }(\boldsymbol{\mu m})\end{array}$ & $\begin{array}{c}\text { FWHM } \\
(\boldsymbol{\mu m})\end{array}$ & Purpose \\
\hline TIR 1 & 8.65 & 0.35 & Temperature/emissivity separation \\
\hline TIR 2 & 9.0 & 0.35 & Temperature/emissivity separation \\
\hline TIR 3 & 10.6 & 0.7 & Split-window \\
\hline TIR 4 & 11.6 & 1.0 & Split-window \\
\hline
\end{tabular}

The targeted launch date for TRISHNA satellite is end of 2024, being then positioned as a precursor of the LSTM Copernicus mission from ESA. TRISHNA is designed for a lifetime of 5 years.

\section{TRISHNA SYSTEM OVERVIEW}

The TRISHNA System and its major components are illustrated by the architecture diagram depicted in Figure 2 showing the repartition of responsibilities between both agencies CNES and ISRO.

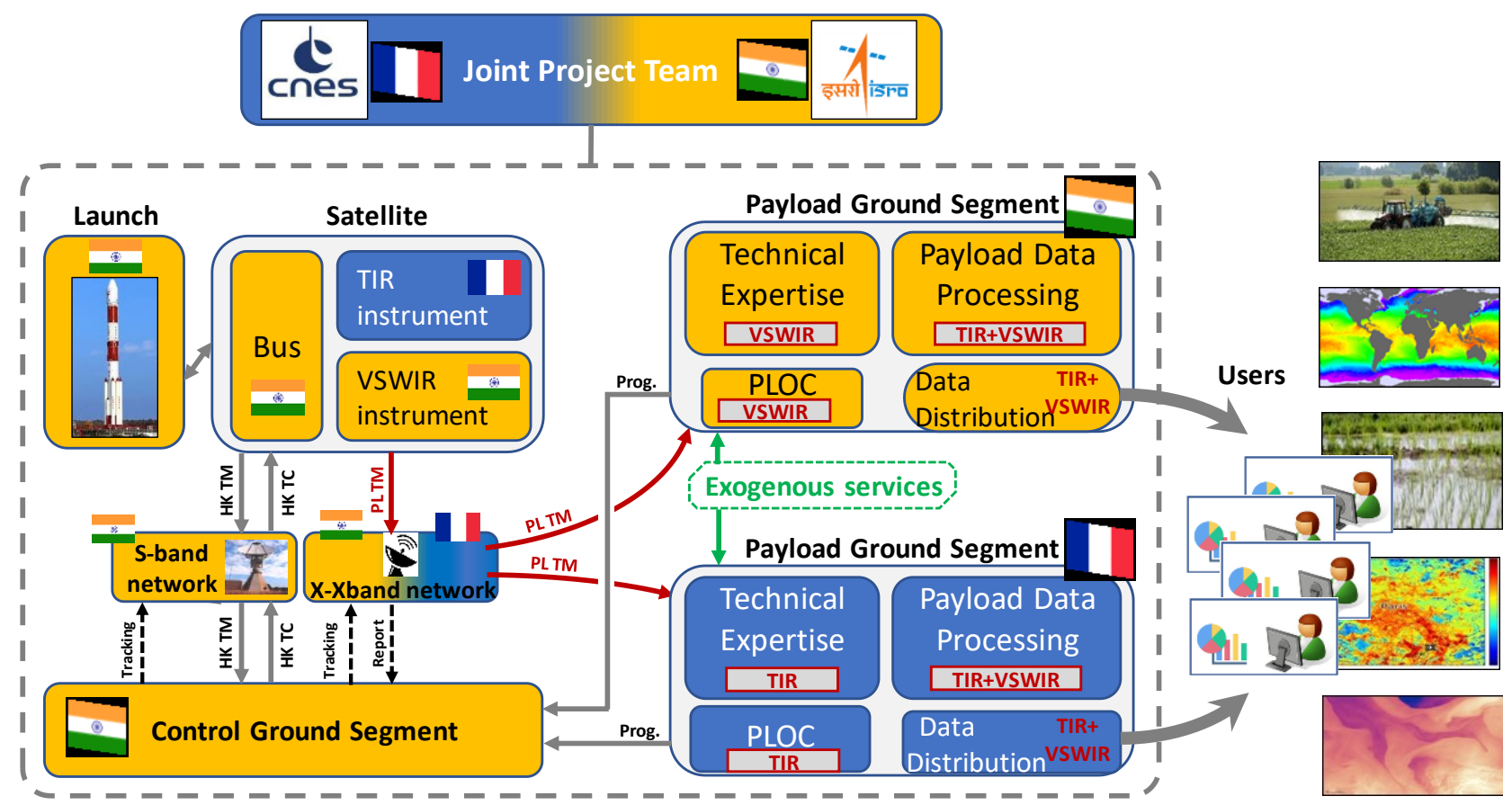

Figure 2. TRISHNA system architecture

Based on this overall architecture, the major components of the TRISHNA System are:

- the space segment composed of:

- a platform, based on IRS-1k, developed and qualified by ISRO.

- the TIR instrument, which remotely senses the planet in the TIR spectrum, developed and qualified by CNES with Airbus Defence and Space as a prime contractor. 
- the VNIR/SWIR instrument, which remotely senses the planet in the VNIR/SWIR spectrum, developed and qualified by ISRO.

- the ground segment composed of:

- a command and control centre for bus and instruments command and control developed and operated by ISRO.

- a support from ISRO S band earth terminals for telecommand and telemetry of the satellite.

- a support from CNES and ISRO X band earth terminals and their connection to the payload ground segment.

- two payload ground segments encompassing:

○ two PayLoad Data Processing (PLDP) chains with the same functionalities, one at CNES and one at ISRO. The PLDP builds up the products from level 0 to level 3, and distributes the products.

- A Payload Operation Centre (PLOC) for instrument command and control.

- Two Technical Expertise Centre (TEC), one at CNES for TIR instrument, one at ISRO for VNIR/SWIR instrument. The TEC are in charge of payload expertise, instrument calibration and characterization, and of the product quality monitoring.

The Launcher and launch services are provided by ISRO.

\section{TIR INSTRUMENT OVERVIEW}

\subsection{Instrument main requirements}

At TIR instrument level, the main requirements are summarized in Table 3 below.

Table 3. TIR main requirements

\section{TIR instrument main requirements}

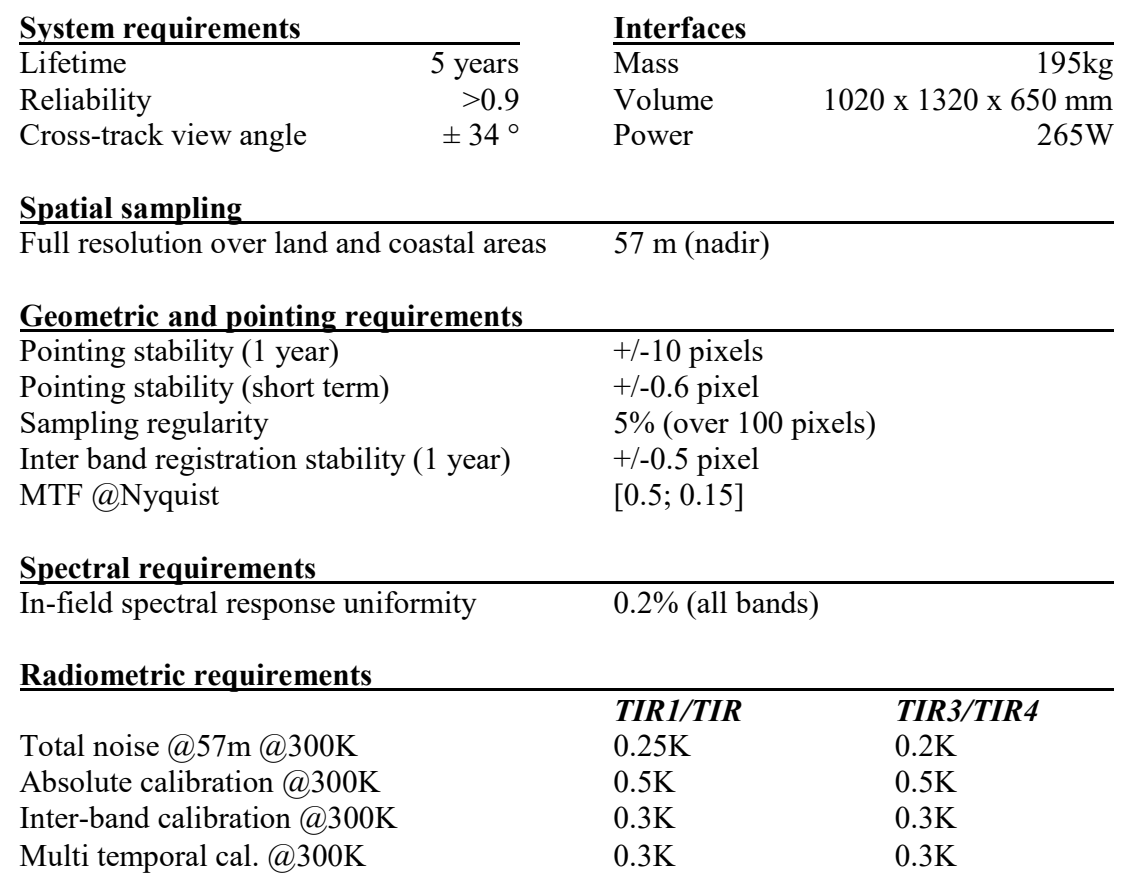




\subsection{Instrument design}

The TIR instrument is based on a scanner concept imposed by the large required swath of $+/-34^{\circ}$. The scan mechanism continuously rotates the entrance plane mirror so that the telescope optical FOV scans the full swath over the so-called Basic Repeat Cycle (BRC). Outside the angular range corresponding to the swath, the scan mirror points successively deep space through a hole in the radiator (for offset calibration) and an internal black-body product (for gain calibration).

The collected optical beam is focused on the focal plane by a Three Mirrors Anastigmatic (TMA) telescope. A field lens just in front of the focal plane guarantees the same beam incidence on each of the four strip filters to avoid spectral decentering effect.

The detector and filters are cooled at $60 \mathrm{~K}$ (nominal working point) through a Cold Thermal Link by two cryocoolers LPT6510 in hot redundancy. This cold optical assembly is thermally insulated from the rest of the instrument by a cryostat using Additive Layer Manufacturing (ALM) technology. The detector outputs data to the TRISHNA Front End Electronic Module (TFEEM) through a flex allowing minimizing thermal heat leaks. ICU controls three downstream electronic modules dedicated to mechanism drive (MDE), Cryocooler Control (CCE), and detector signal read-out and digitalization (TFEEM). It also acquires and controls the Black-body absolute temperature and ensures data processing, and instrument operational thermal control. Both ICU and MDE are redundant. Each of the two CCEs drives a Cryocooler Mechanical Assembly (CMA) in hot redundancy.

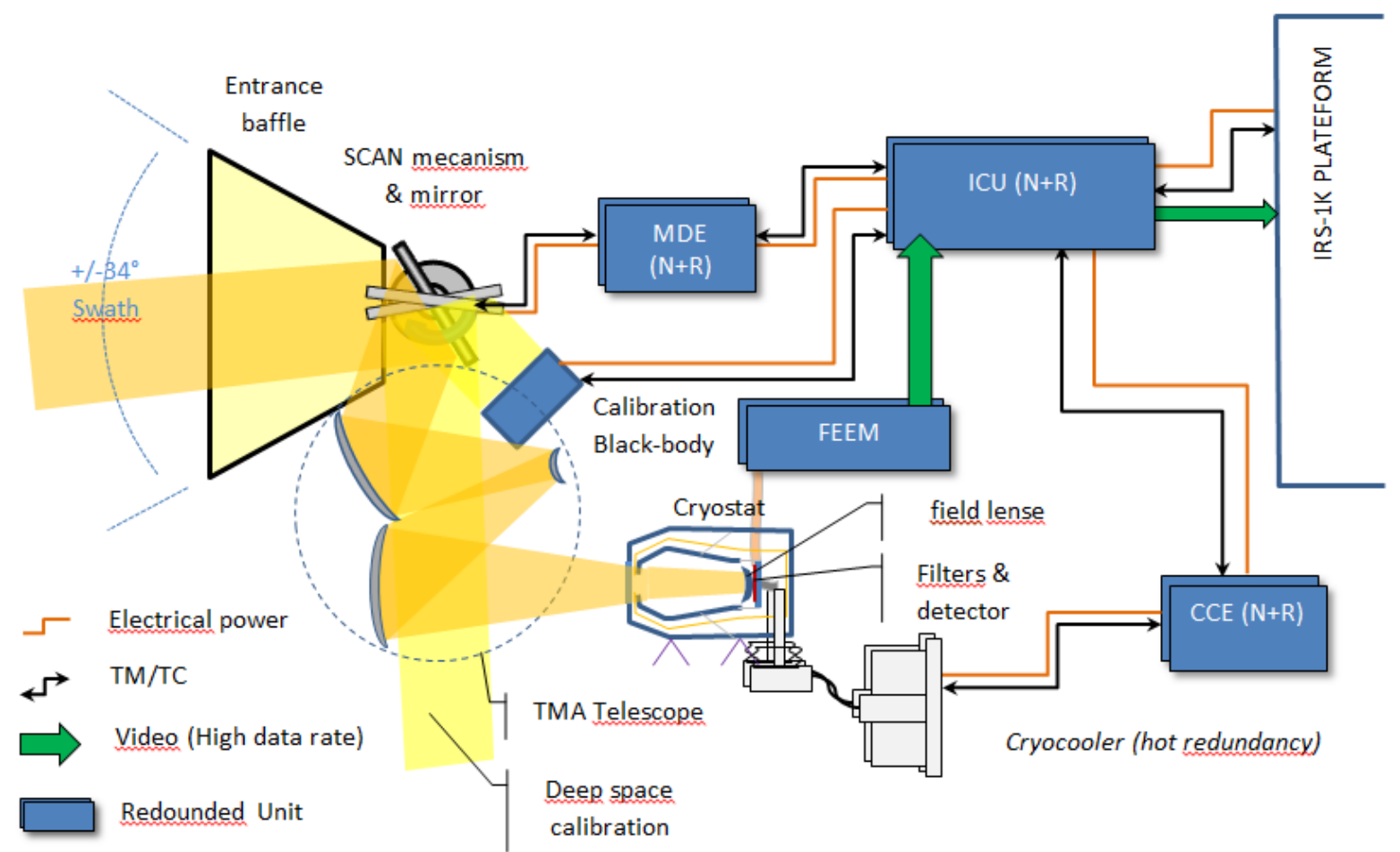

Figure 3. TIR instrument functional overview

\section{Detector}

The LYNRED four-band Mercury-Cadmium-Telluride (MCT) detector features two monoliths with different stoichiometry for SNR optimization: cutoff wavelengths at $10 \mu \mathrm{m}$ (for TIR1 and TIR2) and $12.7 \mu \mathrm{m}$ (for TIR3 and TIR4) at $60 \mathrm{~K}$. The detector pixel size is $30 \mu \mathrm{m}$. For each spectral band, up to 3 TDI stages can be selected, depending on the SNR budget required for each band. In practice the distance between bands is $4 \mathrm{~mm}$ across track. The detector is composed of 600 pixels along track. For each spectral band, each of the three available TDI stages is composed of four pixels, the best one being selected among four.

\section{Optical configuration}

TIR instrument implements a reflective optical configuration favoring general accommodation and stray-light mitigation. Ground Sampling Distance is adapted to the detector $30 \mu \mathrm{m}$ pixel size thanks to a tilted TMA Korsch telescope providing the adequate focal length, an intermediate focal plane and an exit pupil for implementation of a cold stop. The $150 \mathrm{~mm}$ equivalent diameter entrance pupil is fully adapted to the existing blackbody product. A field stop is placed at 
the intermediate focal plane, limiting the stray light levels. The useful beam enters directly the telescope M1 mirror which saves a folding mirror. All mirrors are coated with Gold to optimize the instrument transmission in the useful TIR spectral range. A ZnSe field lens enclosed in the cryostat ensures the same beam incidence on each filter for each detector pixel.

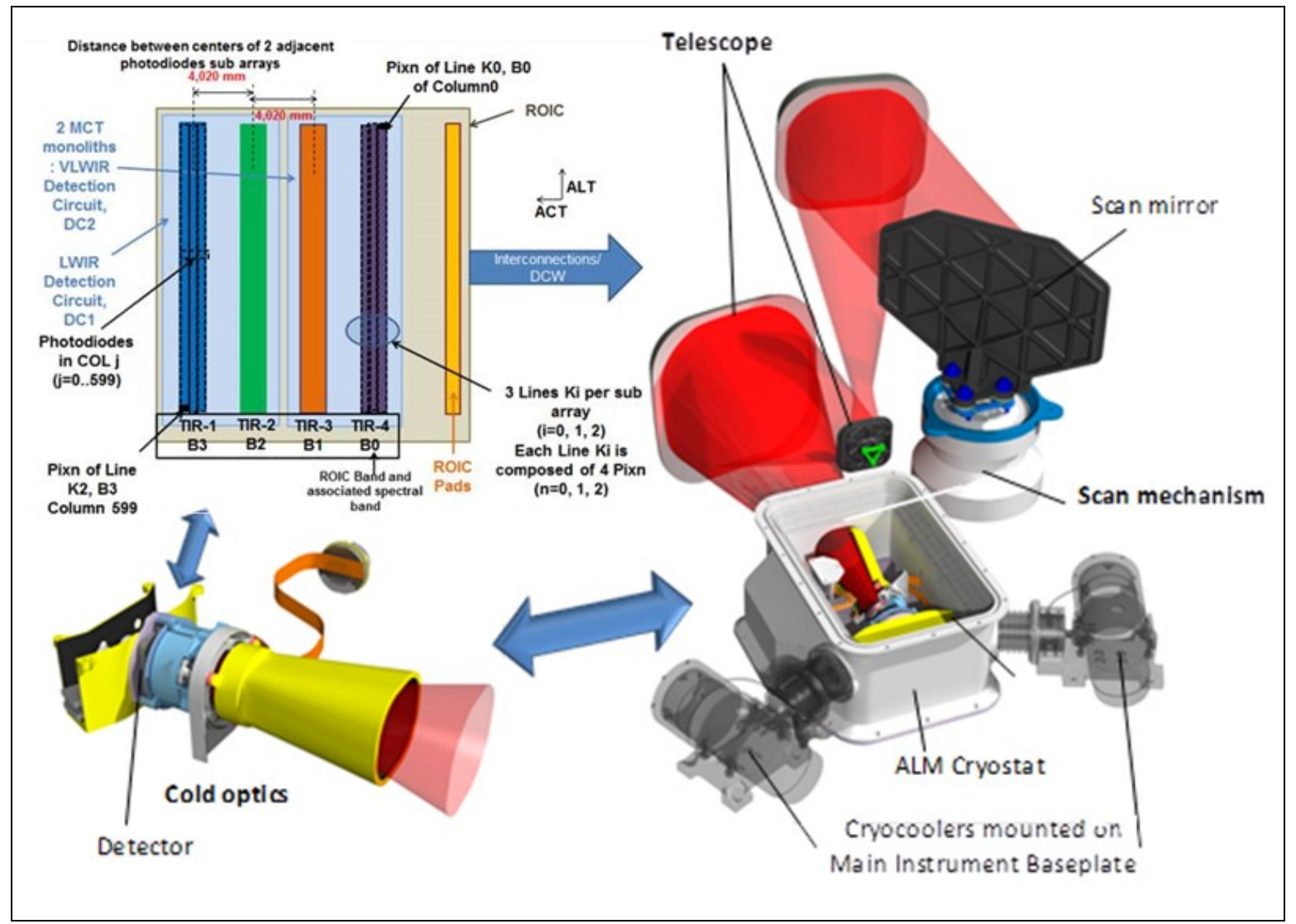

Figure 4. LYNRED detector layout and TMA telescope optical configuration

\section{LYNRED detector ROIC and detection circuit topology with 4 bands of 600 pixels each (up to 3 TDI stages per band) is dedicated to TRISHNA TIR instrument and can be reused for TIR imaging missions.}

\section{Scanning and calibration}

The scan mirror is mounted on a scan mechanism and its drive unit inherited from other program and reused with an adapted control loop. Angular position restitution is done by an encoder. The Basic Repeat Cycle (BRC) baseline duration is around $5.0 \mathrm{sec}$. After swath scan, the mirror continues its rotation journey until black-body angular position passing in front of the deep space calibration port during outward and return journey. Deep space and black-body acquisitions are performed during each BRC after the Earth scene scanning period, so that the calibration table can be updated for each pixel every 5 seconds.

The black-body is passively maintained around $293 \mathrm{~K}$, and the absolute temperature seen by each band of the detector is known with an uncertainty lower than $50 \mathrm{mK}$ (NeTD) thanks to high precision dedicated temperature measurement chain.

\section{A redundant electrical architecture}

Instrument Computer Unit (ICU) powers and controls the different electronic modules, distributes instrument synchronization, acquires black-body absolute temperature. It ensures video data processing including TDI summation for each band and centralizes all the instrument active operational thermal control. In full coherence with Irs- $1 \mathrm{k}$ platform, ICU is totally redundant and materialized by two independent electronic equipments.

Two LPT6510 cryocoolers are operated in hot redundancy in nominal conditions. Each Cryocooler Control Electronic (CCE) powers the associated Cryocooler Mechanical Assembly (CMA), controls the cold finger temperature level and temperature stability. As a consequence, each ICU can operate both CCEs. 


\section{Main mechanical and thermal architecture concepts}

The TIR instrument mechanical architecture is designed as three main sub-systems:

- The interface frame responsible for the instrument mounting via feet bolted along its perimeter on the platform interface plateau.

- The Electronic Equipment module composed of the Main Instrument Baseplate (MIB), designed as a box including the electronic units. The MIB supports also the radiators and two phase thermal hardware. The MIB is mounted on the interface frame via 4 pods insuring TIR instrument mechanical and thermal insulation from the platform.

- The optical module corresponds to the optical bench supporting all optics including detector and cold optics inside the cryostat, the TMA telescope, the scan mirror and mechanism. It is mounted on the MIB by three bipods.

- TIR instrument thermal control is based on:

- MLI insulation from platform on $-X$ sides, and from the external space environment on $+X$ side.

- Electronics thermal control by direct mounting on Main Instrument Baseplate.

- Optical bench insulation by MLI and temperature regulation at $20^{\circ} \mathrm{C}$ to guarantee the LOS stability needs.

$\circ$ Detector and filters cooled at $60 \mathrm{~K}$ in cryostat through cryocoolers in hot redundancy. Detector temperature is maintained at $60 \mathrm{~K}$ even with a single active cryocooler.

○ LPT6510 cold finger and compressor cooled by $+Z$ radiator through heat-pipes.

- Passive thermal control of the front baffle conductively insulated from its mechanical support.

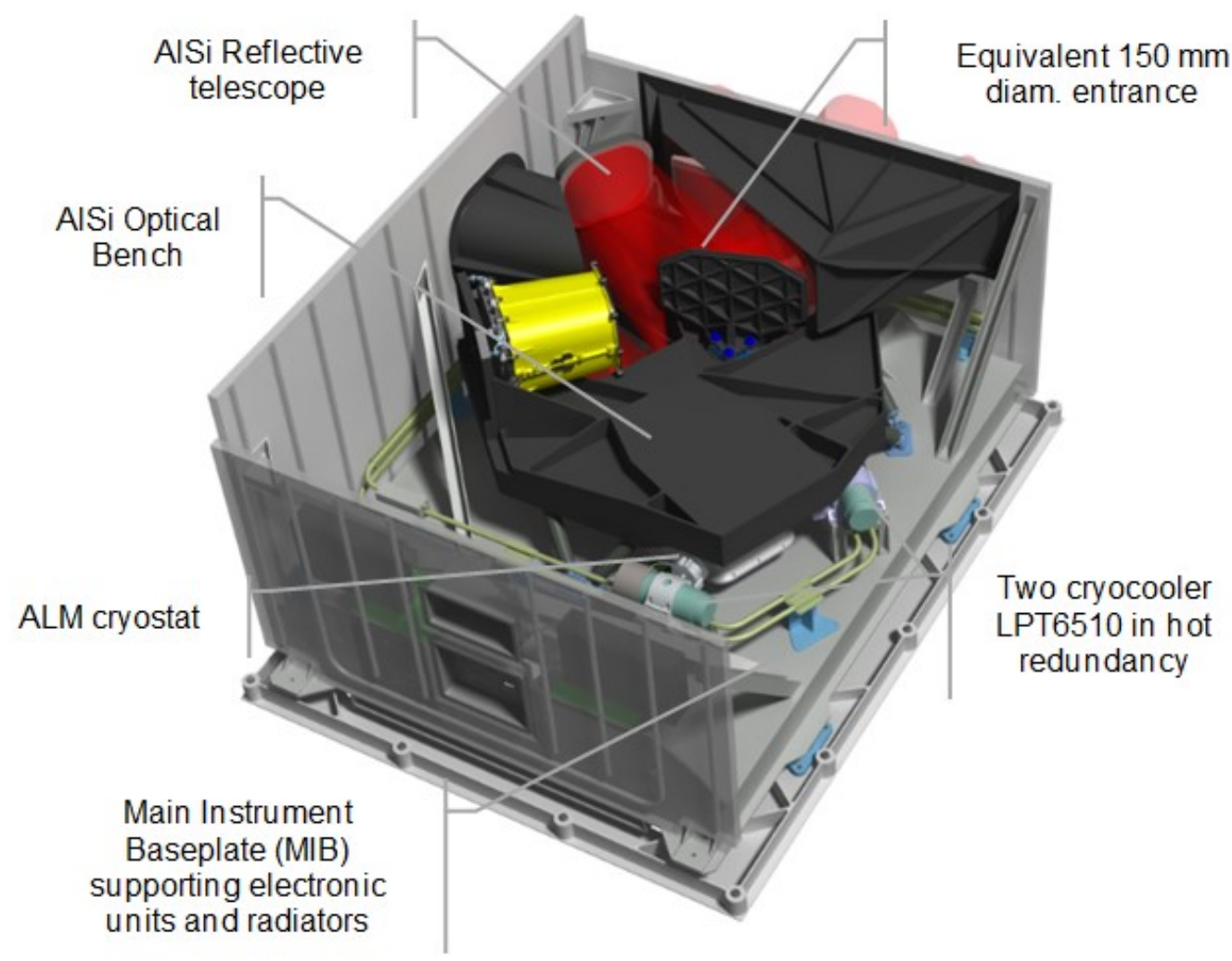

Figure 5. TIR instrument key features 


\section{TECHNOLOGICAL INNOVATIONS}

\subsection{Detector active cooling by LPT6510}

For TRISHNA TIR instrument, Thales Cryogenics B.V. LPT6510 was selected considering two cryocoolers in hot redundancy. Two such cryocoolers provide the necessary cooling power for the required performances at less than mid power at compressor input. LPT6510 still provides the required performance (detector at $60 \mathrm{~K}$ ) in case of a single active cryocooler. The LPT6510 is now being developed and mechanical part of the LPT6510 base product reached TRL6 in 2020.

\section{A cryocooler adapted to the TRISHNA mission}

LPT6510 product is being developed to provide $1 \mathrm{~W}$ at $60 \mathrm{~K}$ with the thermal interface at $20^{\circ} \mathrm{C}$ at end of life. Performances can be increased up to $1.3 \mathrm{~W}$ at $60 \mathrm{~K}$ by cooling the interface down to $0^{\circ} \mathrm{C}$ so this is a point of optimization for TRISHNA TIR instrument. According to TIR instrument cryostat heat-leaks (including uncertainties), the detector can be cooled down to $60 \mathrm{~K}$ with both cryocooler around mid-power.

Taking advantage of the large $+Z$ wall lowest sink temperature, radiator size is designed so as to allow the rejection of the cryocooler dissipation at $0^{\circ} \mathrm{C}$. The parasitic heat leak induced by an off-state LPT6510 is specified under $2 \mathrm{~mW} / \mathrm{K}$. This value is defined from TCBV and Absolut System experience and dedicated measurements on several cold fingers.
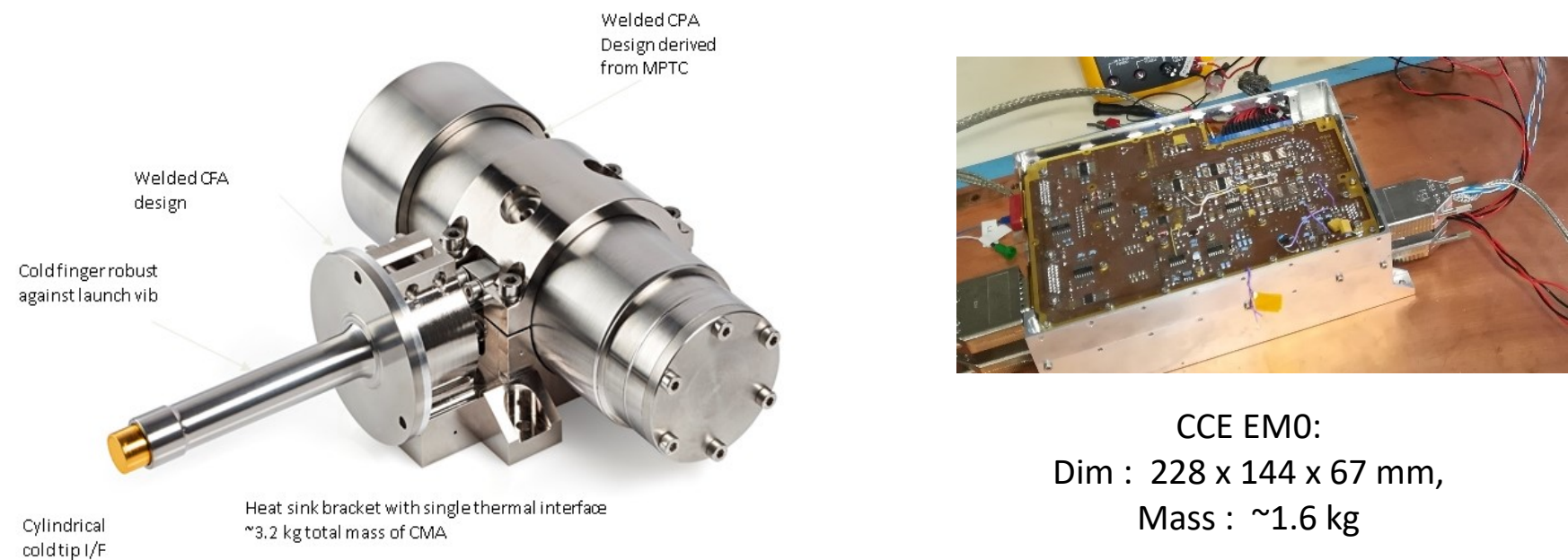

CCE EMO:

$\operatorname{Dim}: 228 \times 144 \times 67 \mathrm{~mm}$, Mass : $1.6 \mathrm{~kg}$

Figure 6. LPT6510 Mechanical Assembly (CMA) EM1 and Drive Electronic (CCE) EM0

\section{Cryocooler failure case working point}

The failure configuration corresponds to the failure of one of the two operating cryocoolers. In this case a heat leak occurs in the OFF-state cold finger corresponding to roughly $400 \mathrm{~mW}$. The remaining cryocooler is then to provide alone the complete heat lift plus the leaks induced by the failed cryocooler. In this condition, the available radiative surface allows cooling of the remaining LPT 6510 at $-15^{\circ} \mathrm{C}$ with its maximum power dissipation which is increased above $60 \mathrm{~W}$ in this case. With the control threshold lowered, the efficiency of the LPT6510 is increased further and leaks through the OFF-state cold finger are lowered. The detector temperature is thus maintained at $60 \mathrm{~K}$ without any impact of the failed cryocooler on the instrument radiometric performances.

\section{Performance growth potential with two operating cryocoolers}

Considering two cryocoolers operating, the large cooling margins allow reduction of the temperature below 60K. Even if the detector working point is $60 \mathrm{~K}$, its temperature can be reduced down to $56 \mathrm{~K}$ (with proper cryocoolers input power increase) which provides additional total noise margin especially for TIR-3 and TIR-4 bands.

\section{A Dutch/French consortium}

LPT6510 is supplied by Thales Cryogenics BV (Eindhoven, Netherlands). TCBV is responsible for the whole Cryocooler Unit, and responsible for Cryocooler Mechanical Assembly (CMA). The CMA is composed of a Compressor Assembly (CPA) developed, qualified and assembled by TCBV and a Cold Finger Assembly (CFA) developed, qualified and assembled by Absolut System (Seyssinet-Pariset, France). The CMA is driven by a CryoCooler driver Electronic (CCE) developed, qualified and produced by STEEL Electronique (Martres Tolosane, France). LPT6510 is then supplied by a well-balanced Dutch/French consortium led by Thales Cryogenics BV (Eindhoven, Netherlands). 


\subsection{A cryostat based on Additive Layer Manufacturing technology}

Thermal insulation of the cold focal plane from its warm environment is the main function of the cryostat. Two options of manufacturing and assembly have been traded-off to answer the need for TRISHNA TIR instrument. The integration of a conventional space cryostat is complex and requires a large number of operations, with more than 40 parts composing the shells and GFRP (Glass Fiber Reinforced Polymer) bipods, as well as SLI (Single Layer Insulation) between shells, increasing integration risks and bringing adsorbed water in the cryostat cold enclosure.

For TRISHNA mission, the selected baseline is a cryostat based on Additive Layer Manufacturing (ALM) as it allows a much simpler design with reduced number of parts and reduced manufacturing and AIT cost, at equivalent thermal insulation performances.

\section{The Additive Layer Manufacturing (ALM) cryostat: an innovative solution reducing AIT planning}

This capability of ALM to manufacture several parts in one allows a drastic reduction of the number of parts compared to conventional technology. This induces a significant simplification of the assembly, as well as justification analyses of the interfaces. Besides there is no more need for the kapton SLIs which are complex to integrate, and the GFRP material can be removed (eliminating the associated risk of moisture and molecular contamination).

Regarding mechanical and thermal performances, the ALM cryostat reaches thermal insulation performance similar to a so-called "conventional space cryostat". Another asset is the flexibility of ALM technology with capability to manufacture complex shapes with internal embedded patterns and junctions, and its reactivity to late design evolutions adding also development planning flexibility.

Thanks to these assets, the AIT duration can be reduced compared to the conventional cryostat solution.

\section{A secured ALM technology development plan}

The maturity level of the ALM cryostat is not as high as the conventional cryostat. A thorough development plan has been set up in order to quickly reduce the associated risks and reach maturity levels adapted to the project schedule. Airbus already qualified ALM flying parts, and during 2020 and first part of 2021, Airbus progresses on de-risking of the technology for ALM manufacturing, local machining of ALM parts and CT scan (X ray tomography) considering preliminary design. Mechanical vibration, cleanliness and thermal performance measurements on samples are also performed to confirm the maturity of the technology.

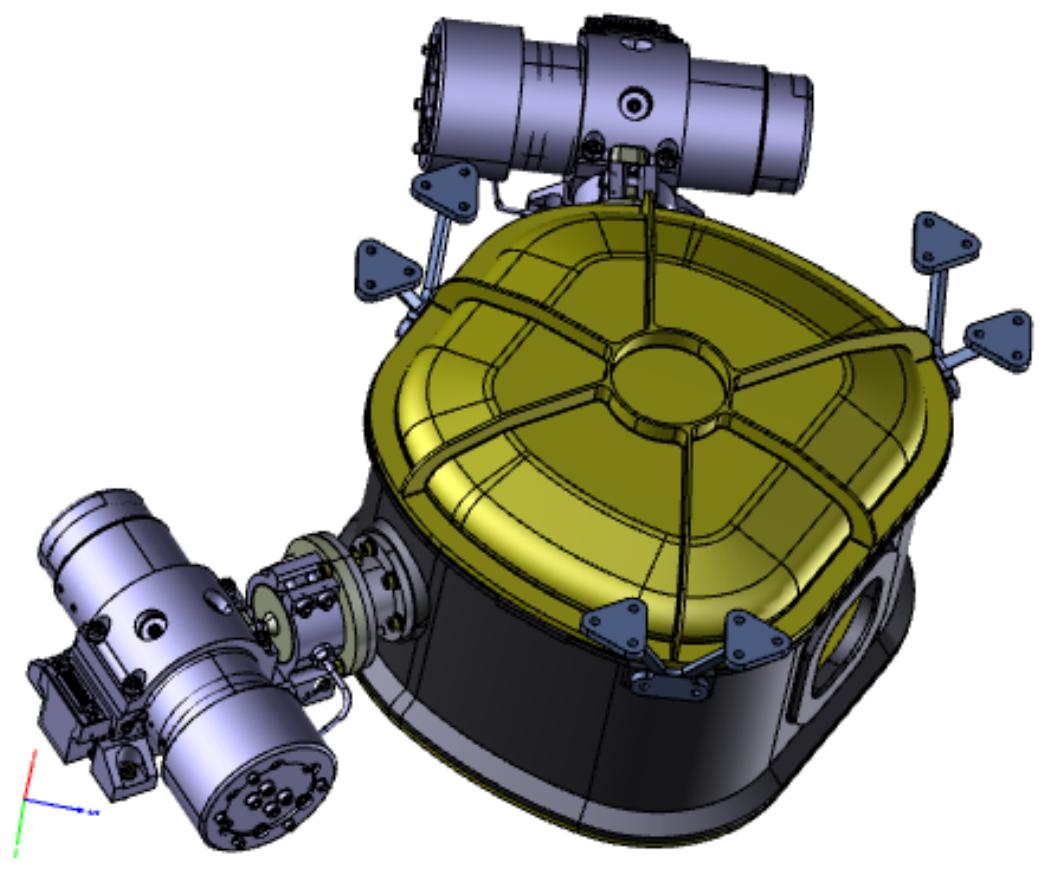

Figure 7. Cryostat housing design overview with LPT6510 cryocooler interfacing

ALM cryostat simplifies assembly integration, replacing more than 40 parts by only a few monolithic parts. The ALM cryostat has been selected as the best solution for TIR cryostat considering cost, planning, performances and risks. ALM is baselined for the manufacturing of the cryostat housing. Conventional assembly of machined part is presented as a backup. 


\section{INSTRUMENT DEVELOPMENT STATUS}

\section{Instrument}

In the context of the TRISHNA mission French/Indian cooperation, CNES chose at the very beginning of 2020 Airbus to develop the TIR instrument. The instrument contract was officially Kicked-off in early June 2020, and a Preliminary Design Review is forecasted as planned in May 2021. The Critical Design Review of this short lead-time program will follow during summer 2022, and the delivery of the TIR instrument in ISRO premises in Bangalore (India) is currently planned mid-2024 for a launch end of 2024.

\section{Detector}

For the detector, a first Read Out Integrated Circuit (ROIC) predevelopment was initiated under CNES lead with a functional test campaign at ambient temperature in order to validate good functionality of the device. Next steps are to validate functionality at operational temperature and to manufacture hybridized retina to be in position to assess detailed detector electro-optical performances, before mounting in the dedicated package and electrical interfaces connection to the Detector Cold Wiring (DCW) via bondings. Overall development schedule takes benefit from the strong heritage provided by the recent METImage program sharing the same detection circuit for the VLWIR bands TIR3\&TIR4 and the associated dedicated electro-optical test benches, but also previous heritage from MTG concerning the detector package and associated DCW and connector design.

\section{LPT6510 Cryocooler}

Cryocooler product qualification was performed by Thales Cryogenics BV between 2019 and 2020 . A specific Equipment Qualification for TRISHNA TIR instrument specific working points is in progress and is expected to be closed in 2021 .

Building blocks of the cryocooler CCE unit were developed by Steel in the frame of the Medium Pulse Tube Cooler (MPTC) under CNES contract. Thanks to these development elements, the CCE design could be anticipated before instrument Kick-off, and an EM was successfully tested at ambient temperature with a LPT6510 Engineering Model (EM), and reached TRL5 by September 2020. This CCE EM demonstrated with a fully flight representative LPT6510 CMA (already at TRL6), the required cold tip temperature stability with margin (in closed loop temperature control mode). CCE design has been adapted for TIR instrument specific launch-locking needs and ICU electrical interfacing is ready for PDR in the first quarter of 2021.

\section{ALM Cryostat}

ALM technology promising for cryostat needs is baselined for TRISHNA and expected to be used for future cold focal planes. On the basis of TRISHNA TIR focal plane interface needs, an EM0 was manufactured in 2020. It demonstrated adequate cleanliness properties. Tests will continue in the first part of 2021 in order to demonstrate maturity of the ALM technology for such an application.

\section{CONCLUSION}

TRISHNA will provide new observation capacities in the thermal infrared domain thanks to its high spatio-temporal resolution data. TRISHNA data will be helpful to a wide range of scientific user communities and will contribute to the development of operational applications for a better management of water resources.

In order to fulfil the required performances, TIR instrument design relies on an optimized solution compatible with a short development schedule. It is based on an assembly of recurring equipments and technological innovations which demonstration is expected to be completed during phase $\mathrm{B}$.

\section{REFERENCES}

[1] LPT6510 Test results up to TRL6, International Cryocooler Conference ICC 21, E. Jansen, R. Arts, J. Mullié, Thales Cryogenics B.V., Eindhoven, The Netherlands, J. Tanchon and T. Trollier, Absolut System SAS, Seyssinet-Pariset, France 